\title{
CALCULUS PROOFS OF SOME COMBINATORIAL INEQUALITIES
}

\author{
TOMISLAV DOŠLIĆ AND DARKO VELJAN
}

\begin{abstract}
Using calculus we show how to prove some combinatorial inequalities of the type log-concavity or log-convexity. It is shown by this method that binomial coefficients and Stirling numbers of the first and second kinds are log-concave, and that Motzkin numbers and secondary structure numbers of rank 1 are log-convex. In fact, we prove via calculus a much stronger result that a natural continuous "patchwork" (i.e. corresponding dynamical systems) of Motzkin numbers and secondary structures recursions are increasing functions. We indicate how to prove asymptotically the log-convexity for general secondary structures. Our method also applies to show that sequences of values of some orthogonal polynomials, and in particular the sequence of central Delannoy numbers, are log-convex.
\end{abstract}

Mathematics subject classification (2000): 05A20, 05A10, 26 A06.

Key words and phrases: log-concavity, log-convexity, Motzkin numbers, Delannoy numbers, secondary structures, Legendre polynomials, calculus.

\section{REFERENCES}

[1] M. Aigner, Motzkin numbers, Europ. J. Combinatorics, 19 (1998), 663-675.

[2] E.A. BENDER AND E.R. CANFIELD, Log-concavity and related properties of the cycle index polynomials, J. Comb. Theory A, 74 (1996), 56-70.

[3] F. BRENTI, Unimodal, log-concave and Polya frequency sequences in combinatorics, American Math. Society, Providence, RI, 1989.

[4] D. CALlan, Notes on Motzkin and Schröder numbers, preprint, 2000.

[5] T. DošLić, Problems of Matching Enumeration and Some Applications to Biochemical Graphs, Ph.D. thesis, University of Zagreb, Zagreb, 2001.

[6] T. DoŠLiĆ, D. SVRTAN AND D. VELJAN, Secondary structures, preprint, 2001.

[7] S. KARLIN, Total positivity, Stanford Univ. Press, Stanford, 1968.

[8] J. Kruskal D. SANKofF, Time Warps, String Edits and Macromolecules, (2nd edition), Adison-Wesley, Reading, 1999.

[9] B. SAGAN, Inductive and injective proofs of log-concavity results, Discr. Math., 68 (1988), 281-292.

[10] R. STANLEY, Log-concave and unimodal sequences in algebra, combinatorics and geometry, Ann. N.Y. Acad. Sci., 576 (1989), 500-535.

[11] R. Stanley, Enumerative Combinatorics, vol. 2, Cambridge Univ. Press, Cambridge, 1999.

[12] R. StANLEY, Positivity problems and conjectures in algebraic combinatorics, in Mathematics: Frontiers and Perspectives, (Eds. V. Arnold et al.), IMU-AMS, 2000, 295-319.

[13] G. SzEGÖ, Orthogonal Polynomials, Amer. Math. Soc., New York, 1959.

[14] M.S. WATERMAn, Secondary structures of single stranded nucleic acids, in G.C. Rota, editor, Advances in Mathematics, Academic Press, New York, 1978. 